\title{
PRECONCENTRACIÓN DE PLOMO EN MUESTRAS SINTÉTICAS POR EXTRACCIÓN CON TRITÓN X-114 EN EL PUNTO DE NUBE Y ANÁLISIS POR ABSORCIÓN ATÓMICA (EAAF)
}

\author{
Marixa Zegarra Pisconti ${ }^{1 *}$ y Jesús Cjuno Huanca ${ }^{2}$
}

\begin{abstract}
RESUMEN
Se desarrolló una metodología de preconcentración de plomo en muestras de agua sobre las que se adicionó ditizona como agente acomplejante previamente disuelto en el surfactante no iónico Tritón X-114 hasta la formación de la concentración micelar crítica y en la temperatura del punto de nube. El centrifugado del sistema dio un precipitado con alta concentración de $\mathrm{Pb}$ (II) la misma que fue cuantificada por espectroscopía de absorción atómica con flama (EAAF). El procedimiento ha demostrado ser viable para ser implementado como un método de preconcentración y análisis de $\mathrm{Pb}$ en muestras acuosas con concentraciones menores a 1 ppm. Se evaluó diversos parámetros obteniéndose un porcentaje de recuperación del 89,8\%.

Palabras clave: Preconcentración, surfactante, ditizona, concentración micelar crítica (CMC), temperatura de punto de nube, Tritón X-114, EAAF, recuperación.

\section{LEAD PRECONCENTRATION IN SYNTHETIC SAMPLES WITH TRITON X-114 IN THE CLOUD POINT EXTRACTION AND ANALYSIS BY ATOMIC ABSORPTION (EAAF)}

\begin{abstract}
A methodology was developed about lead preconcentration in water samples that were added dithizone as complexing agent, previously dissolved in the nonionic surfactant Triton X-114, until the formation of the critical micelle concentration and the cloud point temperature. The centrifuged system gave a precipitate with high concentrations of $\mathrm{Pb}$ (II) that was measured by atomic absorption spectroscopy with flame (EAAF). The method has proved feasible to be implemented as a method of preconcentration and analysis of $\mathrm{Pb}$ in aqueous samples with concentrations less than $1 \mathrm{ppm}$. Several parameters were evaluated to obtain a percentage recovery of $89.8 \%$.
\end{abstract}

Key words: Preconcentration, surfactant, dithizone, critical micelle concentration (CMC), cloud point temperature, Triton X-114, EAAF, recovery.

\section{INTRODUCCIÓN}

El plomo es un metal que se encuentra en la corteza de la tierra en forma de minerales (galena, cerusita, anglesita). Las actividades humanas relacionadas con liberación del plomo en el ambiente son la minería, fundición, refinación y reciclaje informal del plomo, el uso de gasolina con plomo, la producción y el reciclaje de baterías de plomo-ácido y pinturas,

\footnotetext{
1* UPG, Facultad de Química e Ingeniería Química, UNMSM. Av. Universitaria /Av. Germán Amézaga s/n Lima-1, Perú. marixa_zegarra@yahoo.com

2 Departamento Académico de Fisicoquímica, FQIQ, UNMSM.
} 
soldadura, fábrica de cerámica, residuos electrónicos y el uso de plomo en tuberías (antiguas) de agua. En el ambiente, el plomo se puede encontrar en forma de partículas en suspensión en la atmósfera, el agua, el suelo y la biota en general. El plomo se reconoce como un elemento tóxico a nivel mundial.

Los análisis del $\mathrm{Pb}$ se realizan a partir de diversas matrices, como son las provenientes de medios ambientales, biológicos, alimentos, etc.

En el agua, los estándares nacionales de calidad ambiental (ECA - agua) establecen que el nivel máximo permisible para el plomo sea de $0,01 \mathrm{mg} / 1^{1}$, referencias que exigen equipos muy sensibles, no siempre accesibles a todos los laboratorios. Una alternativa a estos inconvenientes son los métodos de preconcentración y separación como por ejemplo las técnicas de intercambio iónico realizadas por los mismos autores en artículos anteriores ${ }^{2,3}$, las de coprecipitación, extracción líquido-líquido, extracción en fase sólida o como en los últimos años la preconcentración y extracción con surfactantes acondicionado a su punto de nube ${ }^{4,5,6,7}$

La separación y preconcentración basada en la extracción con tritón X-114 en el punto de nube (CPE siglas del inglés cloud point extraction), tema del presente artículo, ha llegado a ser una aplicación práctica e importante en el uso de surfactantes en química analítica ${ }^{8,9,10}$. El principio, ventajas y aplicaciones de la técnica han sido bien establecidos en los últimos años ${ }^{11,12}$.

Los surfactantes o sustancias anfifílicas se pueden representar esquemáticamente por la fórmula $\mathrm{L}-\mathrm{H}$. La parte lipofílica de la molécula (L) es en general un radical hidrocarbonado como el dodecilbenceno, por ejemplo. Por otra parte, $\mathrm{H}$ representa la parte hidrofílica o polar de la molécula, que es en general un grupo oxigenado.

Existen numerosos tipos de surfactantes no iónicos, siendo los más comunes los surfactantes etoxilados. En la figura 1 se muestra la estructura del Tritón X-114 (4-octil fenoletoxilato), donde " $n$ " indica el número promedio de unidades de óxido de etileno a un lado de la cadena. Estos son compuestos en los que el grupo hidrofílico es una cadena de poli-oxietileno fijada por la función hidroxilo o amina ${ }^{13}$.

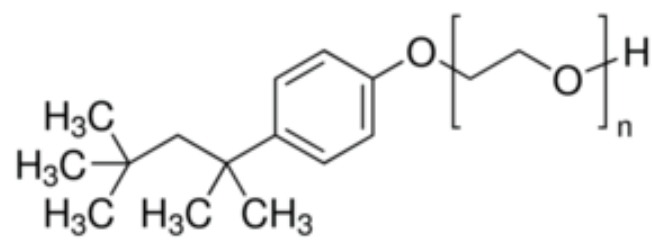

Figura 1. Tritón $\mathrm{X}-114, \mathrm{n}=7-8$ 
Tabla 1. Propiedades del Tritón X-114*

\begin{tabular}{ll}
\multicolumn{1}{c}{ Estado } & Líquido \\
Unidades promedio de óxido de etileno & $7-8$ \\
\% de ingrediente activo & $100 \%$ \\
Color, APHA & 100 \\
Gravedad Específica, $25^{\circ} / 25^{\circ} \mathrm{C}$ & $1.054 \mathrm{~g} / \mathrm{ml}$ \\
Densidad, lb/gal & 8.8 \\
Viscocidad, Brookfield ${ }^{(1)}$, a $25^{\circ} \mathrm{C}, \mathrm{cP}$ & 260 \\
Punto de nube, $1 \%$ solución acuosa, ${ }^{\circ} \mathrm{C}$ & 22 \\
Concentración Crítica Micelar $(\mathrm{CMC})$ & $0.2 \mathrm{mM}$ \\
\hline
\end{tabular}

(1) Husillo \#2 a 12 rpm

* Fuente: http://www4.mpbio.com/ecom/docs/proddata.nsf/(webtds2)/194854

Tabla 2. Solubilidades del Tritón X-114 a temperatura ambiente

\begin{tabular}{lc}
\hline \multicolumn{1}{c}{ Solvente } & Tritón X-114 \\
& $\mathrm{D}$ \\
Agua & $\mathrm{D}$ \\
Soluciones de sales inorgánicas & $\mathrm{S}{ }^{(2)}$ \\
Ácidos minerales & $\mathrm{M}$ \\
$\begin{array}{l}\text { Solventes orgánicos polares (alcoholes, } \\
\text { glicoles, éteres, cetonas, etc.) }\end{array}$ & $\mathrm{M}$ \\
Hidrocarburos aromáticos & $\mathrm{I}$ \\
Hidrocarburos alifáticos & \\
I = Insoluble; D = Dispersable; M = Miscible en todas las proporciones; $\mathrm{S}=$ Soluble \\
l Tritón X-114 es soluble en soluciones diluidas de ácido clorhídrico, fosfórico y sulfúrico
\end{tabular}

En vista de su dualidad polar-apolar, una molécula de surfactante no puede satisfacer su doble afinidad ni en un solvente polar, ni en un solvente orgánico. Cuando una molécula de surfactante se coloca en una interfase agua-aire o agua-aceite, ella puede orientarse de manera que el grupo polar esté en el agua, mientras que el grupo apolar se ubica "fuera" del agua, en el aire o en el aceite. Desde un punto de vista energético, se puede decir que la energía libre de una molécula de surfactante a la interfase es inferior a la de una molécula solubilizada en el seno de una fase acuosa. La transferencia desde el seno de una fase acuosa a la interfase, llamada adsorción es por lo tanto espontánea ${ }^{14}$.

La explicación de M. Fernanda S. et al ${ }^{11}$, en relación a lo dicho anteriormente, es como sigue: "La micela no es más que una zona de exclusión donde aquellas sustancias que no son compatibles con el solvente pueden entrar espontáneamente, esto se conoce como solubilización micelar. 
Se conocen 4 tipos de solubilizaciones: (1) en el corazón de la micela, (2) la cosolubilización, (3) en la superficie de la micela y (4) la solubilización por cadenas de poli-oxietileno. El último tipo de solubilización es característico de las micelas de surfactantes no iónicos, como el Tritón X-114, cuyo hidrófilo consiste en una cadena de polioxietileno. Al parecer, ciertos compuestos orgánicos (como la ditizona) pueden ser secuestrados por estas cadenas hidrofílicas y actúan, por lo tanto, como agentes pseudo-quelantes. Estos agentes pseudoquelantes, pueden formar complejos con determinados tipos de iones metálicos ${ }^{15}$.

Después, la temperatura se eleva (sobre la temperatura de punto de nube) para que ocurra la separación de las fases. La explicación es la siguiente: Cada molécula de surfactante es rodeada por una red de moléculas de agua a través de enlace de puente de hidrógeno en los grupos polares. Cuando la temperatura se eleva, esta red es destruida por la entropía; las débiles fuerzas de Van der Waals prevalecen entre las moléculas del surfactante, originando la separación de fases. Por lo tanto, la separación de las fases resulta de la competencia entre la entropía, la cual favorece la miscibilidad de las micelas en el agua, y la entalpía, la cual favorece la separación de las micelas del agua. El mecanismo por el cual la separación de fases ocurre, se atribuye al rápido incremento del número de agregación de las micelas del surfactante, como resultado del incremento de la temperatura. Los segmentos de óxido de etileno en la micela se repelen entre sí a bajas temperaturas, cuando están hidratadas, y se atraen conforme la temperatura se eleva debido a la deshidratación. Este efecto causa una disminución del área efectiva ocupada por el grupo polar en la superficie de la micela, incrementando el tamaño de la misma que puede ser considerada infinita en el punto de nube, resultando en la separación de las fases.

La turbidez en el sistema surge por la presencia de surfactantes agregados que dispersan la luz visible que pasa a través de la solución.

Finalmente, el paso siguiente es la separación de la fase rica en surfactante, que se ve facilitada por la diferencia de densidades de ambas fases.

\section{Ditizona:}

La ditizona (difeniltiocarbazona) es un reactivo orgánico cuya fórmula molecular es $\mathrm{C}_{6} \mathrm{H}_{5}(\mathrm{NH})_{2} \mathrm{CS}(\mathrm{N})_{2} \mathrm{C}_{6} \mathrm{H}_{5}$; generalmente se le abrevia como $\mathrm{H}_{2} \mathrm{Dz}$. Tiene una masa molecular de $256,33(\mathrm{~g} / \mathrm{mol})$ y tiene la apariencia de cristales color púrpura oscuro.

Se representa como un ácido débil, HL, que pierde un protón cuando se une a un ion metálico a través del azufre y el nitrógeno ${ }^{16}$, tal como se aprecia en la figura 2.

El equilibrio de la ditizona es $^{17}$ :

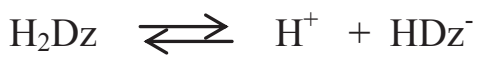

La ditizona es un reactivo utilizado en la determinación de metales debido a que forma complejos coloreados con muchos de ellos. En la figura 2 se muestra la estructura del ditizonato de plomo; se ha demostrado por estudios de rayos X, que la molécula está conformada por dos ligandos bidentados de ditizona coordinados tetraédricamente al ion metálico, tal como se aprecia en la figura 2 . 


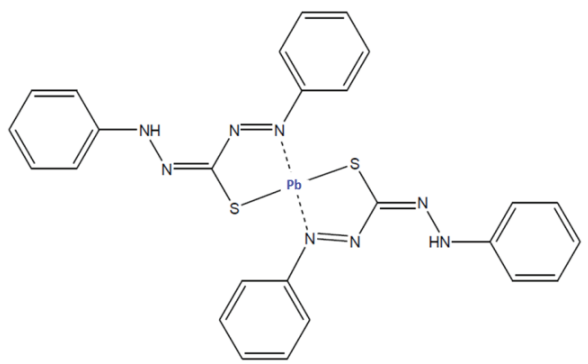

Figura 2. Ditizonato de plomo

La reacción también se puede representar como:

$$
\begin{aligned}
& \left.\mathrm{Pb}^{2+}+\mathrm{H}_{2} \mathrm{O} \quad \longleftrightarrow \quad \mathrm{Pb}(\mathrm{OH})\right]^{+}+\mathrm{H}^{+} \\
& 2 \mathrm{H}_{2} \mathrm{Dz}_{(\mathrm{ac})}+[\mathrm{Pb}(\mathrm{OH})]^{+} \rightleftarrows \mathrm{Pb}(\mathrm{HDz})_{2(\mathrm{ac})}+\mathrm{H}_{3} \mathrm{O}^{+}
\end{aligned}
$$

Por la especie química predominante de acuerdo al diagrama de pourbaix del $\mathrm{Pb}^{18}$.

En el presente artículo presentamos los resultados de aplicar la CPE para la extracción del plomo en agua, utilizando como agente acomplejante la ditizona (1,5 difeniltiocarbazona) y posterior determinación por espectrofotometría de absorción atómica con flama (EAAF).

\section{PARTE EXPERIMENTAL}

Todos los trabajos fueron realizados en el periodo de 2013 al 2014, en el laboratorio de análisis instrumental del Departamento de Minería y Procesos Químico-Metalúrgico Tecsup Nro. 1, concluyéndose en septiembre del 2014.

\section{Equipos:}

- Centrífuga: Fisher, modelo 228

- Conductímetro: Marca: Hanna instruments (resolución $=0,01 \mu \mathrm{S} \mathrm{cm}^{-1}$ )

- $\quad$ HH-metro: Marca: Thermo Scientific-Orion 5 Star (resolución=0,01 pH)

- Plancha de calentamiento eléctrica con agitador magnético (60-1200 rpm), WisestirMSH-20D

- Balanza analítica, Mettler Toledo, capacidad $220 \mathrm{~g}, \mathrm{~d}=0,1 \mathrm{mg}$

- Espectrofotómetro de absorción atómica con flama, Perkin Elmer, Analyst-100. Softaware: winlab 32

- Espectrofotómetro de absorción molecular Thermospectronic, Genesys 10UV

\section{Reactivos:}

- Fosfato diácido de potasio $\left(\mathrm{KH}_{2} \mathrm{PO}_{4}\right)$, Q.P.

- $\quad$ Hidróxido de sodio $(\mathrm{NaOH})$, Q.P.

- Ditizona $\mathrm{C}_{6} \mathrm{H}_{5} \mathrm{NHNHCSN}: \mathrm{NC}_{6} \mathrm{H}_{5}, \mathrm{PM}=256,32$, Spectrum

- Tritón X-114, (Polyethylene glycol tert-octylphenyl ether) Sigma-Aldrich

- Solución estándar de plomo de 1000 ppm. Merck

- Ácido nítrico, $65-70 \%$, J.T. Baker

- Agua desionizada $\left(\mathrm{pH}=5,50, \mathrm{CE}=2,00 \mu \mathrm{S} \mathrm{cm}^{-1}\right)$ 


\section{Procedimiento}

El procedimiento experimental se ha esquematizado en la figura 3
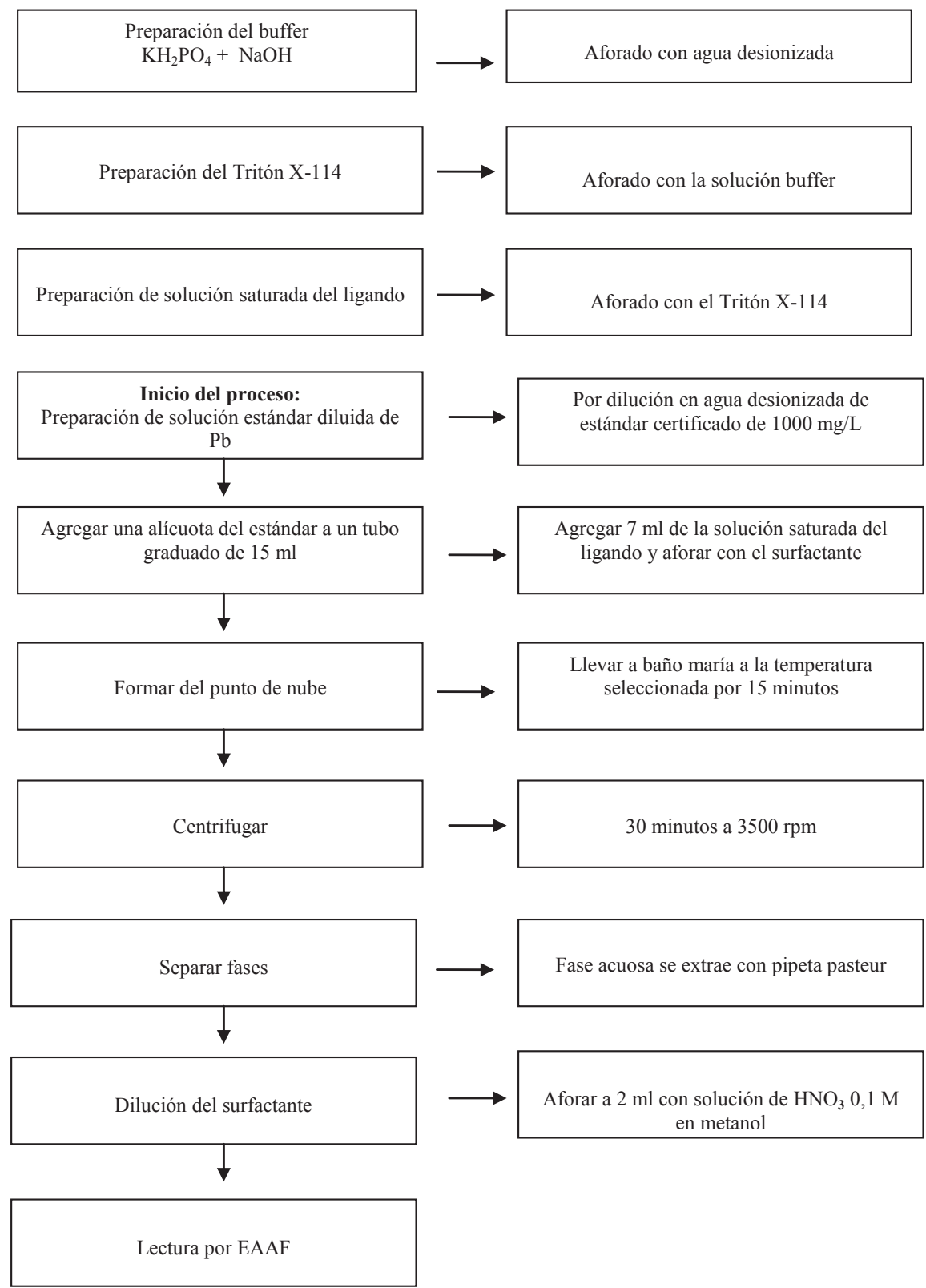

Figura 3. Procedimiento general de la extracción por punto de nube 


\section{Preparación de reactivos:}

- Se preparó buffer fosfato/ $\mathrm{NaOH}$ de $\mathrm{pH}=6,7$ y 8

- Preparación del Tritón X-114 0,2 \% a pH 7: para 100 ml de solución de Tritón X-114 se preparó pesando $0,2000 \mathrm{~g}$ de Tritón $\mathrm{X}-114$ y aforando a $100 \mathrm{ml}$ con el buffer de $\mathrm{pH}=7$. Las demás soluciones de surfactante a diferentes porcentajes de Tritón X-114 y pH, se prepararon, cambiando en cada caso, el peso del surfactante y aforando con el buffer de $\mathrm{pH}$ deseado.

- Preparación de la solución saturada de ditizona en el Tritón X-114 al pH deseado: se pesó $0,0100 \mathrm{~g}$ de ditizona y se disolvió en $50 \mathrm{ml}$ de Tritón X-114 al 0,2\%; se agitó por 10 minutos y se filtró con papel whatman Nro. 42

- Preparación del estándar de $\mathrm{Pb}$ de 10 ppm, se preparó por dilución a partir del estándar de 1000 ppm.

\section{Preparación de la muestra:}

En tubo de prueba graduado de $15 \mathrm{ml}$ se colocó $1 \mathrm{ml}$ del estándar de Pb de 10 ppm, se añadió $7 \mathrm{ml}$ de la solución saturada de ditizona y se aforó con el Tritón X-114 al pH 7 (caso óptimo). Se homogenizó manualmente. Las determinaciones se realizaron por triplicado para evaluar cada variable.

\section{Formación de la micela y separación de fases:}

- Se colocó inmediatamente en baño maría por 15 minutos a $40{ }^{\circ} \mathrm{C}+/-1^{\circ} \mathrm{C}$

- Se llevó a la centrífuga a $3500 \mathrm{rpm}$ por 30 minutos

- Se colocó en la refrigeradora por 10 minutos y después de eliminó la fase acuosa.

\section{Disolución del surfactante y lectura por EAA:}

La muestra se aforó a $2 \mathrm{ml}$ con $\mathrm{HNO}_{3} 0,1 \mathrm{M}$ en metanol, se homogenizó y se realizó la lectura por EAAF. Los parámetros instrumentales fueron constantes para todas las lecturas realizadas: longitud de onda $=283,3 \mathrm{~nm}$, rendija $=0,7 \mathrm{~nm}$, llama oxidante de aire $/ \mathrm{C}_{2} \mathrm{H}_{2}$ con flujos (4:2), cabezal de $10 \mathrm{~cm}$. Se analizó, además, con lámpara de cátodo hueco y nebulizador de alta sensibilidad. Se prepararon estándares de $0,2,5$ y $10 \mathrm{mg} / 1$ en medio de $\mathrm{HNO}_{3} 0,1 \mathrm{M}$ en metanol.

\section{RESULTADOS Y DISCUSIÓN}

En las figuras 4 a la 6 se muestra el porcentaje de recuperación cuando se ha variado las condiciones de $\mathrm{pH}$, concentración del surfactante y temperatura. En todos los casos se añadió $1 \mathrm{ml}$ de solución estándar de $\mathrm{Pb}$ de 10ppm.

1. pH:

En la figura 4 se muestra el resultado del porcentaje de recuperación del plomo añadido a diferentes soluciones amortiguadas de $\mathrm{pH}$. Los análisis se realizaron por triplicado. 


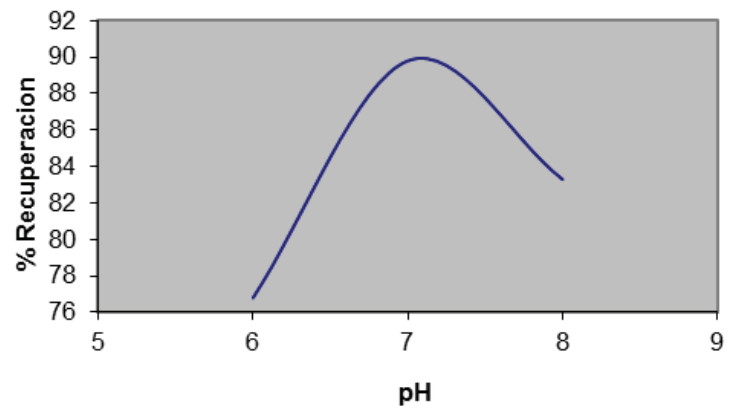

Figura 4. Porcentaje de recuperación a diferentes medidas de $\mathrm{pH}$

Se obtuvo un máximo porcentaje de recuperación a $\mathrm{pH}=7,00$, a $\mathrm{pH}$ menores a 7 se aprecia una disminución del porcentaje de recuperación (76,8\%) debido al desplazamiento del equilibrio de la reacción hacia la izquierda, lo que desestabiliza el complejo (ecuación 3).

\section{Porcentaje de Tritón X-114:}

En la figura 5 se muestra el resultado del porcentaje de recuperación del plomo añadido a soluciones con diferente concentración de surfactante. Los análisis se realizaron por triplicado.

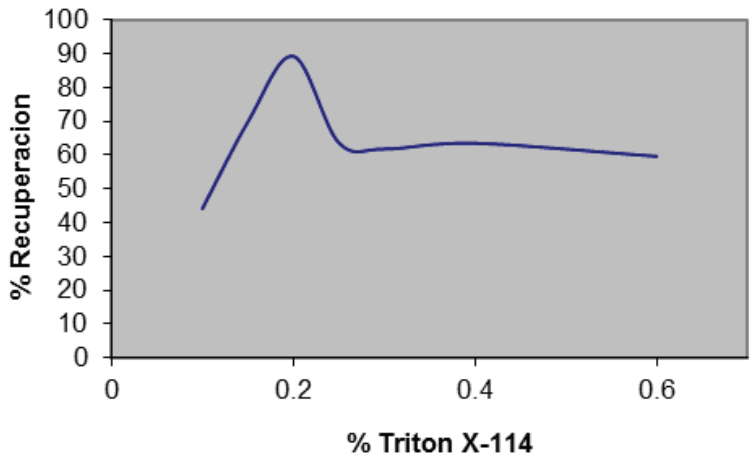

Figura 5. Porcentaje de recuperación a diferentes \% del surfactante

Como se observa en la figura 5, se obtiene una recuperación máxima con una concentración de Tritón X-114 del 0,2\%; a concentraciones inferiores el porcentaje de recuperación disminuye; es decir, la eficiencia de preconcentración del complejo baja debido, probablemente, a la inhabilidad de atrapar el complejo hidrofóbico, ya que no hay cantidad suficiente de micelas para "atrapar" al complejo. A concentraciones superiores de surfactante hay un incremento del volumen de la fase rica en surfactante. Algunos autores consideran ${ }^{19}$ que la viscosidad de esta fase origina bajas recuperaciones.

\section{Temperatura:}

En la figura 6 se muestra el resultado del porcentaje de recuperación del plomo añadido a soluciones con diferentes temperaturas. Los análisis se realizaron por triplicado. 


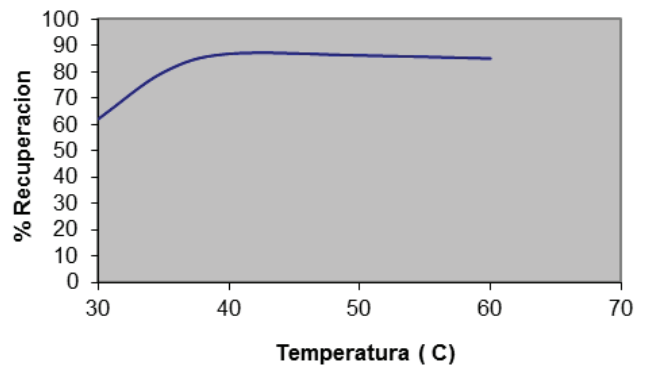

Figura 6. Porcentaje de recuperación a diferentes temperaturas

En la figura 6 se observa que hay un incremento moderado en la solubilización hasta los 40 ${ }^{\circ} \mathrm{C}$; presumiblemente esto refleje un incremento en la agitación térmica de las moléculas del surfactante en las micelas. Esto tiende a incrementar el número de agregación y/o tamaño de las micelas. Luego disminuye la cantidad de analito solubilizado conforme se va elevando la temperatura; esto, como lo mencionan algunas publicaciones ${ }^{15,20}$, causa un incremento de la deshidratación del oxietileno en el grupo hidrofílico; o lo que es lo mismo, la desolvatacion de las cadenas de polióxido de etileno y como consecuencia la reducción del carácter hidrofílico del surfactante y las cadenas del surfactante que se unen dejando poco espacio disponible en las capas.

En todos los casos se trabajó con solución saturada de $\mathrm{H}_{2}$ Dz para asegurar un acomplejamiento completo del metal. En este trabajo se encontró que la solubilidad de la ditizona en TX-114 al $0,2 \%$ a $\mathrm{pH}=7$ fue de $0,018 \mathrm{~g} / \mathrm{l}$, cuyo valor fue hallado experimentalmente siguiendo el procedimiento seguido por Rajesh P. Paradakar y Ron R. Williams ${ }^{21}$

En las pruebas de recuperación los mayores valores se obtuvieron centrifugando a una velocidad de $3500 \mathrm{rpm}$ por 30 minutos.

En todas las pruebas la separación de fases se vio favorecida por refrigeración a $4{ }^{\circ} \mathrm{C}$ por 15 minutos.

Para las lecturas por EAAF, al complejo extraído en el Tritón X-114 se le añadió $\mathrm{HNO}_{3} 0,1$ $\mathrm{M}$ en metanol, obteniendo un volumen final de $2 \mathrm{ml}$.

Los parámetros instrumentales del equipo de absorción atómica, se eligieron, para cada curva de calibración, para mantener la linealidad de las mismas ${ }^{22}$.

El cálculo del porcentaje de recuperación del plomo añadido al surfactante, se obtuvo multiplicando la concentración hallada por lectura directa de las soluciones de plomo en la solución metanólica, dividiendo entre la concentración añadida y multiplicando por 100. El porcentaje del plomo recuperado del surfactante se calculó de acuerdo a la siguiente fórmula:

$$
\begin{gathered}
\% \operatorname{Rec}=\frac{\left(C_{2} \times F_{c} \times F_{d}\right)}{C_{1}} 100 \\
\% \operatorname{Rec}=\frac{(4,463 \mathrm{mg} / 1 \times 0,2)}{(1 \mathrm{mg} / \mathrm{l})} 100=89,26 \%
\end{gathered}
$$

$\mathrm{C}_{2}$ (concentración hallada del promedio de tres lecturas directas en la solución metanólica) $=4,463 \mathrm{mg} / 1$

Fc (factor de concentración) $=0,2$ 


\section{CONCLUSIONES}

La extracción en el punto de nube ofrece ciertas ventajas sobre la extracción tradicional con solventes. Un pequeño volumen de fase rica en surfactante permite no sólo preconcentrar y extraer el analito en un solo paso sino también alcanzar porcentajes de recuperación considerables, como en este caso, cercanos al $90 \%$.

La preconcentración basada en CPE ha mostrado ser una alternativa a los sistemas de extracción tradicionales, permitiendo el diseño de procedimientos de preconcentración accesibles a cualquier laboratorio básico. Es relevante considerar que esta técnica asegura una baja toxicidad por su medio acuoso, comparada con sistemas que utilizan solventes orgánicos en la etapa de preconcentración.

\section{AGRADECIMIENTO}

Al Ing. Qco. Hernán Zapata Gamarra por elaborar el diagrama de pourbaix del plomo y proporcionar la información correspondiente.

\section{BIBLIOGRAFÍA}

1. DS-Nº02-2008-MINAM. Estándares Nacionales de Calidad Ambiental para Agua

2. J. Cjuno, M. Zegarra. Preconcentración de Zn(II) con el intercambiador catiónico PSDVB- $\mathrm{SO}_{3} \mathrm{H}$ con monitoreo de $\mathrm{pH}$ y análisis de recuperación de los eluidos por absorción atómica (EAAF). Rev.Per.Quím.Ing.Quím. 2012; 15(1):15-20

3. M. Zegarra, J. Cjuno. Preconcentración de $\mathrm{Cu}(\mathrm{II})$ con el intercambiador catiónico PSDVB- $\mathrm{SO}_{3} \mathrm{H}$ en un proceso por lotes y análisis de la recuperación por absorción atómica (EAAF). Rev.Per.Quím.Ing.Quím. 2013; 16(1):33-38.

4. Maria das Grac, A. Korna, Jailson B. de Andrade A, Djane S. de Jesus. Procedimientos de separación y preconcentración del plomo usando técnicas espectrométricas. Talanta 2006;69:16-24

5. http://www.intechopen.com/books/atomic-absorptionspectroscopy/microextractiontechniques-as-a-sample-preparation-step-for-metal-analysis (último acceso 8 de septiembre del 2014).

6. Faheem Shah, Tasneem Gul Kazi, Naeem Ullah. Determination of lead in biological samples of children with different physiological Consequences using cloud point extraction method. Biol Trace Elem Res 2013; 153: 134-140

7. Xiadong Wen, Qingwen Deng, Shoulian Ji, Shengchun Yan, Li Peng. Design of rapidily synergistic cloud point extraction of ultra-trace lead combined with flame atomic absorption spectrometry determination. Microchemical journal 2012; 100: 31-35

8. N. Dallali_, M.M. Zahedi and Y. Yamini. Simultaneous cloud point extraction and determination of $\mathrm{Zn}, \mathrm{Co}, \mathrm{Ni}$ and $\mathrm{Pb}$ by flame atomic absorption spectrometry, using 2-guanidinobenzimidazole as the complexing agent. Scientia Iranica, 2007; 14(4): 291296.

9. Daniel L. Gallindo B., Márcia A. Mesquita S., Vera L. Azzolin F., Bernhard Welz and Adilson J. Curtius.Cloud-point extraction for the determination of $\mathrm{Cd}, \mathrm{Pb}$ and $\mathrm{Pd}$ in blood by electrothermal atomic absorption spectrometry, using Ir or Ru as permanent modifiers. J. Anal. At. Spectrom., 2003; 18: 501-507.

10. Mohammad-Hadi Givianrad, Kambiz Larijani, y otros. Assesment of heavy metals by 
ligand-less cloud point extraction in sediment and Holothuria parva (Echinodermata, Holothuroidea). Indian Journal of Geo-Marine Sciences. 2014; 43(5): 825-830

11. María Fernanda Silva, Estela Soledad Cerutti, Luis Martínez. Coupling Cloud Point Extraction to Instrumental Detection Systems for Metal Analysis. Microchim Acta. 2006; $155: 349-364$

12. Constantine D. Stalikas. Micelle-Mediated extraction as a tool for separation and preconcentration $n$ metal analysis. Trends in Analytical Chemistry. 2002; 21(5): $343-$ 355

13. Jean-Louis Salager. Cuaderno FIRP S201-A, Surfactantes en solución acuosa. MéridaVenezuela. Versión \# 2 (1993)

14. Jean-Louis Salager. Cuaderno FIRP S311-A, El Mundo de los Surfactantes. MéridaVenezuela. Versión \# 01 (1992)

15. Rita M. Avila-Gomez- José L. Burguera-Jean-Louis Salager-Carlos L. Bracho. La Micelas en Química Analítica, aplicaciones en la espectroscopía de absorción atómica. Revista de la Sociedad Venezolana de Química. 2003; 26(2): 23-31.

16. Daniel C. Harris. "Análisis químico cuantitativo", tercera edición, Editorial Reverté, Barcelona, España, 2007, 551.

17. E. B. Sandell "Colorimetric Determination of Traces of Metals", tercera edición, Editorial Interscience Publisher, 1959, 144.

18. Outokumpu HSC Chemistry for Windows Chemical Reaction and Equilibrium Software with Extensive Thermochemical Database [Programa informático] versión 6.0 [Finland, 2006]

19. Farshid Ahmadi, Azadeh Khanmohammadi. Micelle mediated methodology for the determination of heavy metals in real samples by flame atomic absorption spectrometry. Kuwait J.SCI.Eng. 2010; 37(1A): 85-98,

20. Rosen, Milton. Surfactants and Interfacial phenomena, Editorial John Wiley \& Son, New York, 2004, 189

21. Rajesh P. Paradakar y Ron R. Williams. Micellar Colorimetric Determination of Dithizone metal Chelates. Anal. Chem. 1994; 66: 2752-2756.

22. APHA-3111-B- 2012. American Public Health Association 